\title{
A SEQUENCE OF ZERO MODES OF WEYL-DIRAC OPERATORS AND AN ASSOCIATED SEQUENCE OF SOLVABLE POLYNOMIALS
}

\author{
Dedicated to Professor Edmunds and Professor Evans \\ on the occasion of their eightieth and seventieth birthdays \\ Yoshimi Saitō ${ }^{\dagger}$ and Tomio Umeda $a^{\ddagger}$ \\ ${ }^{\dagger}$ Department of Mathematics, University of Alabama at Birmingham \\ Birmingham, AL 35294, USA \\ $\ddagger$ Department of Mathematical Sciences, University of Hyogo \\ Himeji 671-2201, Japan \\ †saito@math.uab.edu \\ ‡umeda@sci.u-hyogo.ac.jp
}

\begin{abstract}
It is shown that a series of solvable polynomials is attached to the series of zero modes constructed by Adam, Muratori and Nash [1].

Keywords: Weyl-Dirac operators, magnetic potentials, zero modes, solvable polynomials.

Mathematics Subject Classification 2000: 35Q40, 35P99, 11R09
\end{abstract}

\section{Introduction}

The aim of this note is to point out an interesting and unpredictable connection between zero modes and solvable polynomials. We shall precisely explain our aim.

To this end, we first introduce a Weyl-Dirac operator

$$
H_{A}=\sigma \cdot(D-A)=\sum_{k=1}^{3} \sigma_{k}\left(D_{k}-A_{k}(x)\right),
$$

\footnotetext{
$\ddagger$ Supported by Grant-in-Aid for Scientific Research (C) No. 21540193, Japan Society for the Promotion of Science.
} 
where $\sigma=\left(\sigma_{1}, \sigma_{2}, \sigma_{3}\right)$ is the triple of $2 \times 2$ Pauli matrices

$$
\sigma_{1}=\left(\begin{array}{ll}
0 & 1 \\
1 & 0
\end{array}\right), \quad \sigma_{2}=\left(\begin{array}{cc}
0 & -i \\
i & 0
\end{array}\right), \quad \sigma_{3}=\left(\begin{array}{cc}
1 & 0 \\
0 & -1
\end{array}\right),
$$

$A(x)=\left(A_{1}(x), A_{2}(x), A_{3}(x)\right)$ is a vector potential, and

$$
D=-i \nabla=\left(-i \frac{\partial}{\partial x_{1}},-i \frac{\partial}{\partial x_{2}},-i \frac{\partial}{\partial x_{3}}\right) .
$$

If each component of the vector potential $A$ is a bounded measureable function, then the operator $\sigma \cdot A$ is a bounded self-adjoint operator in the Hilbert space $\mathcal{L}^{2}:=\left[L^{2}\left(\mathbb{R}^{3}\right)\right]^{2}$. Hence it is straightforward that the Weyl-Dirac operator $H_{A}$ defines the unique selfadjoint realization in $\mathcal{L}^{2}$ and its domain is given as $\operatorname{Dom}\left(H_{A}\right)=\mathcal{H}^{1}:=\left[H^{1}\left(\mathbb{R}^{3}\right)\right]^{2}$ whenever $A_{j} \in L^{\infty}\left(\mathbb{R}^{3}\right)$. Here $H^{1}\left(\mathbb{R}^{3}\right)$ denotes the Sobolev space of order 1 .

Definition 1.1. If $\psi \in \operatorname{Ker}\left(H_{A}\right)$, then $\psi$ is called a zero mode of $H_{A}$. In other words, $\psi$ is said to be a zero mode if and only if $\psi \in \operatorname{Dom}\left(H_{A}\right)$ and $H_{A} \psi=0$.

We should remark that the Weyl-Dirac operator is intimately related with the Pauli operator

$$
P_{A}=\sum_{j=1}^{3}\left(D_{j}-A_{j}\right)^{2}-\sigma \cdot B,
$$

where $B$ denotes the magnetic field given by $B=\nabla \times A$. This is because

$$
P_{A}=\{\sigma \cdot(D-A)\}^{2}=H_{A}^{2}
$$

in a formal sense. Roughly speaking, we can say that $\psi$ is a zero mode of the Weyl-Dirac operator $H_{A}$ if and only if it is a zero mode of the Pauli operator $P_{A}$.

It is now well understood that the existence of magnetic fields which give rise to zero modes of the Weyl-Dirac operators has significant implications in mathematics and physics (see [1], [2], [3], 44, [5], 6], [7, [9], 10], [11, [12, [13, [14, [15], [16], [17]). However, Balinsky and Evans [4, 5, 6] and Elton [10] showed that the set of vector potentials which yield zero modes is scarce in a certain sense.

Vector potentials which give rise to zero modes do exist. The first examples of such vector potentials were given by Loss and Yau [13]. Later Adam, Muratori and Nash [1, 2, 3] and Elton [9] constructed further examples of zero modes, using and developing the ideas from [13]. The works [11] by Erdös and Solovej generalize all these examples.

The basic idea of Loss and Yau [13] is to find a solution of the Loss-Yau equation

$$
(\sigma \cdot D) \psi(x)=h(x) \psi(x),
$$

where $h$ is a given (real-valued) function, and then to define a vector potential $A$ so that $\psi$ satisfies the equation $\sigma \cdot(D-A) \psi=0$. Precise statement of their idea is the following. 
Proposition 1.2. (Loss-Yau [13]). Let $\psi \in \mathcal{H}^{1}$ be a solution to the Loss-Yau equation (1.2) with a real valued bounded function $h$. Then $\psi$ is a zero mode of the Weyl-Dirac operator $H_{A}$ with the vector potential defined by

$$
A(x)=\frac{h(x)}{|\psi(x)|^{2}}\left(\psi(x) \cdot \sigma_{1} \psi(x), \psi \cdot \sigma_{2} \psi(x), \psi \cdot \sigma_{3} \psi(x)\right),
$$

where, for $a={ }^{t}\left(a_{1}, a_{2}\right), b={ }^{t}\left(b_{1}, b_{2}\right) \in \mathbb{C}^{2}, a \cdot b$ denotes the inner product:

$$
a \cdot b=\bar{a}_{1} b_{1}+\bar{a}_{2} b_{2} .
$$

In [13], choosing

$$
\psi(x)=\langle x\rangle^{-3}\left(I_{2}+i \sigma \cdot x\right) \phi_{0} \quad\left(\langle x\rangle=\sqrt{1+|x|^{2}}\right),
$$

where $I_{2}$ is a $2 \times 2$ unit matrix and $\phi_{0} \in \mathbb{C}^{2}$ a unit vector, they showed that $\psi$ defined by (1.4) satisfies the Loss-Yau equation (1.2) with

$$
h(x)=\frac{3}{\langle x\rangle^{2}} .
$$

It follows from (1.3) and (1.4) that

$$
A(x)=3\langle x\rangle^{-4}\left\{\left(1-|x|^{2}\right) w_{0}+2\left(w_{0} \cdot x\right) x+2 w_{0} \times x\right\},
$$

where

$$
\left.w_{0}=\phi_{0} \cdot\left(\sigma \phi_{0}\right):=\left(\phi_{0} \cdot\left(\sigma_{1} \phi_{0}\right), \phi_{0} \cdot\left(\sigma_{2} \phi_{0}\right), \phi_{0} \cdot \sigma_{3} \phi_{0}\right)\right),
$$

and $w_{0} \cdot x$ and $w_{0} \times x$ denote the inner product and the exterior product respectively. Proposition 1.2 implies that $\psi$ defined by (1.4) is a zero mode of the Weyl-Dirac operator $H_{A}$ with the vector potential (1.6).

Adam, Muratori and Nash [1] exploited the idea of Proposition 1.2, and successfully constructed a series $\left\{\psi^{(m)}\right\}_{m=1}^{\infty}$, each of which satisfies the Loss-Yau equation (1.2) with

$$
h^{(m)}(x)=\frac{2 m+3}{\langle x\rangle^{2}} \quad(m=1,2, \cdots) .
$$

It is obvious that each $\psi^{(m)}$ is a zero mode of the Weyl-Dirac operator $H_{A^{(m)}}$ with the vector potential

$$
A^{(m)}(x)=\frac{h^{(m)}(x)}{\left|\psi^{(m)}(x)\right|^{2}}\left(\psi^{(m)}(x) \cdot \sigma_{1} \psi^{(m)}(x), \psi^{(m)} \cdot \sigma_{2} \psi^{(m)}(x), \psi^{(m)} \cdot \sigma_{3} \psi^{(m)}(x)\right) .
$$

The goal of this note is to show that a polynomial $P_{m}(t)$ of degree $m+1$ is associated with each zero mode $\psi^{(m)}$ in such a way that the polynomial equation $P_{m}(t)=0$ is solvable and all of the roots of this equation determine a set of zero modes, one of which is designated as $\psi^{(m)}$. Obviously, as $m$ gets larger, it will become more difficult to solve the equation $P_{m}(t)=0$. It is well-known [18] that "there is no formula for the roots of a fifth (or higher) degree polynomial equation in terms of the coefficients of the 
polynomial, using only the usual algebraic operations (addition, subtraction, multiplication, division) and application of radicals (square roots, cube roots, etc)." Here are the first six equations of $P_{m}(t)=0$ :

$$
\begin{aligned}
& P_{1}(t)=0 \Longleftrightarrow 9 t^{2}-34 t+25=0, \\
& P_{2}(t)=0 \Longleftrightarrow 81 t^{3}-747 t^{2}+1891 t-1225=0, \\
& P_{3}(t)=0 \Longleftrightarrow 81 t^{4}-1476 t^{3}+8614 t^{2}-18244 t+11025=0, \\
& P_{4}(t)=0 \Longleftrightarrow 729 t^{5}-23085 t^{4}+256122 t^{3}-1206490 t^{2} \\
& +2306749 t-1334025=0, \\
& P_{5}(t)=0 \Longleftrightarrow 6561 t^{6}-330966 t^{5}+6206463 t^{4}-54143028 t^{3} \\
& +224657551 t^{2}-401846806 t+225450225=0, \\
& P_{6}(t)=0 \Longleftrightarrow 6561 t^{7}-494991 t^{6}+14480613 t^{5}-209304603 t^{4}+1578233251 t^{3} \\
& -6018285581 t^{2}+10271620375 t-5636255625=0 .
\end{aligned}
$$

It is incredable to see that all these polynomial equations are solvable. Actually, by computer-aided calculation we see that

$$
\begin{aligned}
& P_{1}(t)=0 \Longleftrightarrow t=1, \frac{25}{9} \\
& P_{2}(t)=0 \Longleftrightarrow t=1, \frac{25}{9}, \frac{49}{9}, \\
& P_{3}(t)=0 \Longleftrightarrow t=1, \frac{25}{9}, \frac{49}{9}, 9, \\
& P_{4}(t)=0 \Longleftrightarrow t=1, \frac{25}{9}, \frac{49}{9}, 9, \frac{121}{9}, \\
& P_{5}(t)=0 \Longleftrightarrow t=1, \frac{25}{9}, \frac{49}{9}, 9, \frac{121}{9}, \frac{169}{9}, \\
& P_{6}(t)=0 \Longleftrightarrow t=1, \frac{25}{9}, \frac{49}{9}, 9, \frac{121}{9}, \frac{169}{9}, 25 .
\end{aligned}
$$

Based on this observation, it is natural to predict that the roots of the equation $P_{m}(t)=$ 0 must be given by

$$
1,\left(\frac{5}{3}\right)^{2},\left(\frac{7}{3}\right)^{2}, \cdots,\left(\frac{2 m+3}{3}\right)^{2}
$$

for every $m \in \mathbb{N}$. This prediction will be proven to be true in $\$ 4$, though we should like to mention that these polynomials $P_{m}(t)$ will be only implicitly defined in a rather messy manner; see the formula $\left(L_{m}\right)$ in Proposition 2.1 as well as Proposition 2.2 in 92. In relation with this, we emphasize that the bigger $m$ gets, the more complicated $P_{m}(t)$ becomes, as can be seen from $P_{1}(t), \cdots, P_{6}(t)$ above. 
We should like to call $P_{m}(t)$ in their monic forms the Adam-Muratori-Nash polynomials. We feel solvability of $P_{m}(t)$ seems an interesting subject from the view point of Galois theory (see Edwards [8]), though it is well beyond the scope of the present note.

\section{Recurrence formulae}

In this section we follow the line of the arguments demonstrated in Adam-MuratoriNash [1]. For this reason, we shall use the same notation as in [1] to indicate $I_{2}$ and $i \sigma \cdot x$ in the rest of this note; namely

$$
\mathbf{1}=I_{2}, \quad \mathbf{X}=i \sigma \cdot x
$$

Their construction of the zero modes is based on the following ansatz:

$$
\psi^{(m)}(x)=\langle x\rangle^{-(3+2 m)}\left[\left(\sum_{n=0}^{m} a_{n}|x|^{2 n}\right) \mathbf{1}+\left(\sum_{n=0}^{m} b_{n}|x|^{2 n}\right) \mathbf{X}\right] \phi_{0},
$$

where $\langle x\rangle=\sqrt{1+|x|^{2}}$, and $\phi_{0}={ }^{t}(1,0)$. We are going to study the case where $h(x)$ in (1.2) is $\langle x\rangle^{-2}$ multiplied by a constant $\alpha$. By a simple but tedious computation we have

Proposition 2.1. Let $\psi^{(m)}(x)$ be as above with $a_{0}=1$.

(i) Then, for each $m=1,2,3, \cdots$, we have

$$
\begin{aligned}
&(\sigma \cdot D) \psi^{(m)} \\
&=\langle x\rangle^{-(5+2 m)} \sum_{n=0}^{m}\left[(2 m+3) b_{n}|x|^{2 n}\right.\left.-(2 m-2 n) b_{n}|x|^{2(n+1)}\right] \mathbf{1} \phi_{0} \\
&+\langle x\rangle^{-(5+2 m)}\left[\sum_{n=0}^{m-1}\left\{(3+2 m) a_{n}-2(n+1) a_{n+1}\right\}|x|^{2 n}\right. \\
&\left.\quad+(3+2 m) a_{m}|x|^{2 m}-\sum_{n=1}^{m-1} 2(n+1) a_{n+1}|x|^{2(n+1)}\right] \mathbf{X} \phi_{0} .
\end{aligned}
$$

(ii) The equation

$$
(\sigma \cdot D) \psi^{(m)}(x)=\frac{\alpha}{\langle x\rangle^{2}} \psi^{(m)}(x)
$$


where $\alpha$ is a constant, is equivalent to the system

$$
\left(L_{m}\right)\left\{\begin{aligned}
(2 j-1): & 2 j a_{j}-(2 m+5-2 j) a_{j-1}=-3 b_{0} b_{j-1} \\
(2 k): & \left(1 \leq j \leq m, a_{0}=1\right), \\
& (2 k+3) b_{k}-(2 m+2-2 k) b_{k-1}=3 b_{0} a_{k} \\
(2 m+1): & a_{m}=b_{0} b_{m}
\end{aligned}\right.
$$

of $(2 m+1)$ equations for the $(2 m+1)$ unknowns $a_{j}(1 \leq j \leq m)$ and $b_{k}(0 \leq k \leq m)$, where the constant $\alpha$ turns out to be $3 b_{0}$.

It is easy to see that $\left\{a_{n}\right\}_{n=1}^{m}$ and $\left\{b_{n}\right\}_{n=1}^{m}$ can be expressed by $b_{0}$ and the last equation $a_{m}=b_{0} b_{m}$ becomes a polynomial equation for unknown $b_{0}$.

Proposition 2.2. The last equation $(2 m+1)$ of $\left(L_{m}\right): a_{m}=b_{0} b_{m}$ takes the form $P_{m}\left(b_{0}^{2}\right)=0$, where $P_{m}(t)$ is a polynomial of degree $m+1$.

Proof. Since $a_{0}=1$, the first equation $(1)$ of $\left(L_{m}\right)$ is given as $2 a_{1}-(2 m+3)=-3 b_{0}^{2}$. Hence $a_{1}=p_{1}\left(b_{0}^{2}\right)$, where $p_{1}(t)=2^{-1}\{(2 m+3)-3 t\}$. From the second equation (2) of $\left(L_{m}\right)$ we see that $b_{1}$ takes the form $b_{1}=b_{0} q_{1}\left(b_{0}^{2}\right)$, where $q_{1}(t)=(10)^{-1}(10 m+9-9 t)$. Then, by induction, one can show that $a_{j}, 1 \leq j \leq m$, and $b_{k}, 1 \leq k \leq m$, are expressed as $a_{j}=p_{j}\left(b_{0}^{2}\right)$ and $b_{k}=b_{0} q_{k}\left(b_{0}^{2}\right)$ with polynomial $p_{j}(t)$ of degree $j$ and polynomial $q_{k}(t)$ of degree $k$. Thus the equation $b_{0} b_{m}-a_{m}=0$ becomes a polynomial equation $P_{m}\left(b_{0}^{2}\right)=0$, where $P_{m}(t)$ is a polynomial of $t$ of degree $m+1$.

Remark 2.3. The case $m=1$ is discussed in [1]. In this case

$$
\psi^{(1)}(x)=\langle x\rangle^{-5}\left\{\left(1-\frac{5}{3}|x|^{2}\right) \mathbf{1}+\left(\frac{5}{3}-|x|^{2}\right) \mathbf{X}\right\} \phi_{0} .
$$

Proposition 2.4. Let $m$ be a fixed nonnegative integer and let $a_{n}$ and $b_{n}$ be the coefficients in (2.2) with $a_{0}=1$. Then we have

$$
\left(\begin{array}{c}
a_{j} \\
b_{j}
\end{array}\right)=K_{j} K_{j-1} \cdots K_{2}\left(\begin{array}{c}
a_{1} \\
b_{1}
\end{array}\right) \quad(j=2,3, \cdots, m),
$$

where

$$
K_{p}=\left(\begin{array}{cc}
\frac{2 m+5-2 p}{2 p} & -\frac{3 b_{0}}{2 p} \\
\frac{3(2 m+5-2 p) b_{0}}{2 p(2 p+3)} & \frac{2 p(2 m+2-2 p)-9 b_{0}^{2}}{2 p(2 p+3)}
\end{array}\right)
$$


for $p=2,3, \cdots, m$, and

$$
\left(\begin{array}{c}
a_{1} \\
b_{1}
\end{array}\right)=\left(\begin{array}{c}
\frac{2 m+3-3 b_{0}^{2}}{2} \\
\frac{b_{0}}{10}\left(10 m+9-9 b_{0}^{2}\right)
\end{array}\right) .
$$

Proof. We divide the proof into three steps.

(I) Let $2 \leq j \leq m$. It follows from the equation $(2 k)$ in the system $\left(L_{m}\right)$ with $k$ replaced by $j$ that

$$
(2 j+3) b_{j}-(2 m+2-2 j) b_{j-1}=3 b_{0} a_{j}
$$

From the equation $(2 j-1)$ in $\left(L_{m}\right)$ we have

$$
2 j a_{j}-(2 m+5-2 j) a_{j-1}=-3 b_{0} b_{j-1},
$$

or

$$
a_{j}=\frac{(2 m+5-2 j) a_{j-1}-3 b_{0} b_{j-1}}{2 j} .
$$

Then we obtain from (2.9) and (2.10)

$$
\begin{aligned}
(2 j+3) b_{j}-(2 m+2-2 j) & b_{j-1} \\
& =\frac{\left.3 b_{0}\left[(2 m+5-2 j) a_{j-1}-3 b_{0} b_{j-1}\right)\right]}{2 j},
\end{aligned}
$$

or

$$
\begin{aligned}
& (2 j+3) b_{j} \\
& \quad=\frac{3 b_{0}(2 m+5-2 j)}{2 j} a_{j-1}+\frac{2 j(2 m+2-2 j)-9 b_{0}^{2}}{2 j} b_{j-1} .
\end{aligned}
$$

(II) From the equation $(2 j-1)$ in $\left(L_{m}\right)$ with $j=1$ we see that, by noting that $a_{0}=1$,

$$
2 a_{1}-(2 m+3)=-3 b_{0}^{2},
$$

and hence

$$
a_{1}=\frac{2 m+3-3 b_{0}^{2}}{2} .
$$

We have from the equation $(2 k)$ in $\left(L_{m}\right)$ with $k=1$

$$
5 b_{1}-(2 m+2-2) b_{0}=3 b_{0} a_{1},
$$

which is combined with (2.12) to yield

$$
5 b_{1}-2 m b_{0}=\frac{3 b_{0}\left(2 m+3-3 b_{0}^{2}\right)}{2},
$$

or

$$
b_{1}=\frac{b_{0}\left(10 m+9-9 b_{0}^{2}\right)}{10} .
$$


(III) It follows from (2.10) and (2.11) that

$$
\left(\begin{array}{c}
a_{j} \\
b_{j}
\end{array}\right)=K_{j}\left(\begin{array}{c}
a_{j-1} \\
a_{j-1}
\end{array}\right) \quad(j=2,3, \cdots, m)
$$

with $K_{j}$ given by (2.7) with $p$ replaced by $j$. By using (2.14) repeatedly we can obtain (2.6). As for $a_{1}$ and $b_{1},(2.8)$ is justified by (2.12) and (2.13).

Remark 2.5. Proposition 2.4 was used to construct a Maple program to find the the polynomial $P_{m}(t)$ as well as to solve the polynomial equation $P_{m}(t)=0$. We have been able to handle the equations with the Maple program up to the case $m=26$. The first six equations were listed up at the end of $\S 1$.

\section{Monotonicity of the sequence $\left\{\mathcal{R}_{m}\right\}_{m=1}^{\infty}$}

We begin by

Definition 3.1. For each $m \in \mathbb{N}, \mathcal{R}_{m}$ is defined to be the set of all the roots of the polynomial equation $P_{m}(t)=0$, namely,

$$
\mathcal{R}_{m}:=\left\{t \in \mathbb{C} \mid P_{m}(t)=0\right\} .
$$

Proposition 3.2. Let $\mathcal{R}_{m}$ be as above. Then we have

$$
\mathcal{R}_{1} \subset \mathcal{R}_{2} \subset \cdots \subset \mathcal{R}_{m} \subset \cdots,
$$

i.e., the sequence $\left\{\mathcal{R}_{m}\right\}_{m=1}^{\infty}$ is increasing with $m$.

Proof. For $m=1,2,3, \cdots$, let $\psi^{(m)}(x)$ be given by (2.2) and suppose that the coefficients of $\psi^{(m)}(x)$ satisfy the system $\left(L_{m}\right)$, i.e., $\psi^{(m)}(x)$ is a solution to the equation (2.4) and hence a zero mode of the Weyl-Dirac operator $H_{A^{(m)}}$. Thus $b_{0}^{2} \in \mathcal{R}_{m}$.

We now rewrite $\psi^{(m)}(x)$ as

$$
\begin{aligned}
\psi^{(m)}(x) & =\langle x\rangle^{-(3+2(m+1))}\left[\langle x\rangle^{2}\left(\sum_{n=0}^{m} a_{n}|x|^{2 n}\right) \mathbf{1}+\langle x\rangle^{2}\left(\sum_{n=0}^{m} b_{n}|x|^{2 n}\right) \mathbf{X}\right] \phi_{0} \\
& =: \widetilde{\psi}^{(m+1)}(x) .
\end{aligned}
$$

By using the definition $\langle x\rangle^{2}=1+|x|^{2}$, we obtain

$$
\widetilde{\psi}^{(m+1)}(x)=\langle x\rangle^{-(3+2(m+1))}\left[\left(\sum_{n=0}^{m+1} \widetilde{a}_{n}|x|^{2 n}\right) \mathbf{1}+\left(\sum_{n=0}^{m+1} \widetilde{b}_{n}|x|^{2 n}\right) \mathbf{X}\right] \phi_{0},
$$


where

$$
\left\{\begin{array}{l}
\widetilde{a}_{0}=a_{0}=1, \\
\widetilde{a}_{n}=a_{n-1}+a_{n} \quad(1 \leq n \leq m), \\
\widetilde{a}_{m+1}=a_{m}, \\
\widetilde{b}_{0}=b_{0}, \\
\widetilde{b}_{n}=b_{n-1}+b_{n} \quad(1 \leq n \leq m), \\
\widetilde{b}_{m+1}=b_{m} .
\end{array}\right.
$$

Therefore, noting that $\psi^{(m)}(x)$ satisfies the equation

$$
(\sigma \cdot D) \psi^{(m)}(x)=3 b_{0}\langle x\rangle^{-2} \psi^{(m)}(x)
$$

and that $\widetilde{b}_{0}=b_{0}$ by (3.5) , we see that

$$
\begin{aligned}
(\sigma \cdot D) \widetilde{\psi}^{(m+1)}(x) & =(\sigma \cdot D) \psi^{(m)}(x) \\
& =3 b_{0}\langle x\rangle^{-2} \psi^{(m)}(x) \\
& =3 \widetilde{b}_{0}\langle x\rangle^{-2} \widetilde{\psi}^{(m+1)}(x) .
\end{aligned}
$$

Thus, since $\widetilde{\psi}^{(m+1)}(x)$ is a zero mode, we see that the coefficients $\widetilde{a}_{n}$ and $\widetilde{b}_{n}$ satisfy the system $\left(L_{m+1}\right)$ which is $\left(L_{m}\right)$ in Proposition 2.1(ii) with $m$ replaced by $m+1$. Thus we have the system of $(2 \mathrm{~m}+3)$ equations for

$$
\begin{cases}(2 j-1) & 2 j \widetilde{a}_{j}-(2 m+7-2 j) \widetilde{a}_{j-1}=-3 \widetilde{b}_{0} \widetilde{b}_{j-1} \\ (2 k) & \left(1 \leq j \leq m+1, \widetilde{a}_{0}=1\right), \\ & (2 k+3) \widetilde{b}_{k}-(2 m+4-2 k) \widetilde{b}_{k-1}=3 \widetilde{b}_{0} \widetilde{a}_{k} \\ (2 m+3) & \quad(1 \leq k \leq m+1), \\ \widetilde{a}_{m+1}=\widetilde{b}_{0} \widetilde{b}_{m+1} . & \end{cases}
$$

Therefore $b_{0}=\widetilde{b}_{0}$ satisfies the polynomial equation $P_{m+1}\left(b_{0}^{2}\right)=0$.

Remark 3.3. Proposition 3.2 above does not give us enough information to determine the set $\mathcal{R}_{m}$ though it significantly clarifies the situation. In fact, we know that

$$
\mathcal{R}_{m}=\left\{1,\left(\frac{5}{3}\right)^{2},\left(\frac{7}{3}\right)^{2}, \cdots,\left(\frac{2 m+3}{3}\right)^{2}\right\}
$$

for $m=1, \cdots, 6$. We also know that the polynomial $P_{m}(t)$ is of degree $m+1$. Therefore Proposition [3.2, together with these two facts, tells us that we can prove (3.7) by induction on $m$. In fact, assuming that (3.7) with $m$ replaced by $m-1$ is true, we only have to show that

$$
\left(\frac{2 m+3}{3}\right)^{2} \in \mathcal{R}_{m}
$$

for every $m \geq 2$ (actually $m \geq 7$ ). 


\section{Construction of zero modes}

We are going to prove that (3.7) is true for every $m \in \mathbb{N}$, and describe how to construct the sequence $\left\{\psi^{(m)}\right\}_{m=1}^{\infty}$ of zero modes in terms of a root of the polynomial equation $P_{m}\left(b_{0}^{2}\right)=0$.

Recall that in Proposition 2.2 we saw that $a_{j}=p_{j}\left(b_{0}^{2}\right)$ and $b_{k}=b_{0} q_{k}\left(b_{0}^{2}\right)$ with the polynomials $p_{j}(t)$ and $q_{k}(t)$ of degrees $j$ and $k$ respectively, where $1 \leq j, k \leq m$. Let

$$
\left\{\begin{array}{l}
c_{j}:=p_{j}(0) \\
d_{k}:=\lim _{t \rightarrow \infty} \frac{q_{k}(t)}{t^{k}} .
\end{array}\right.
$$

In other words, $c_{j}$ denotes the constant coefficient of the polynomial $p_{j}(t)$ and $d_{k}$ denotes the coefficient of $t^{k}$ in the polynomial $q_{k}(t)$, of which degree is $k$.

Lemma 4.1. Let $m$ be a fixed nonnegative integer and let $a_{n}$ and $b_{n}$ be the coefficients in (2.2) with $a_{0}=1$. Then we have

$$
c_{m}=\frac{5 \cdot 7 \cdot 9 \cdots(2 m+3)}{2^{m}(m !)}
$$

and

$$
d_{m}=(-1)^{m} \frac{3^{2 m}}{5 \cdot 7 \cdot 9 \cdots(2 m+3) 2^{m}(m !)} .
$$

Proof. We divide the proof into two steps.

(I) From the equation $(2 j-1)$ of $\left(L_{m}\right)$ we have

$$
2 j a_{j}-(2 m+5-2 j) a_{j-1}=-3 b_{0} b_{j-1},
$$

where the right hand side has no constant coefficient as a polynomial of $b_{0}$. Hence we have

$$
2 j c_{j}-(2 m+5-2 j) c_{j-1}=0 .
$$

Therefore we obtain recursive relations

$$
c_{j}=\frac{2 m+5-2 j}{2 j} c_{j-1} \quad(j=2, \cdots, m),
$$

which implies that

$$
c_{j}=\frac{(2 m+5-2 j)(2 m+5-2(j-1)) \cdots(2 m+1)(2 m+3)}{2^{j}(j !)},
$$

where we should note that $c_{1}=(2 m+3) / 2$. We obtain (4.2) by setting $j=m$ in (4.5).

(II) Let $\widetilde{c}_{j}$ be the coefficient of $t^{j}$ of $p_{j}(t)$. Then it follows from the equation $(2 j-1)$ of $\left(L_{m}\right)$ that

$$
2 j \widetilde{c}_{j}=-3 d_{j-1}
$$

or

$$
\widetilde{c}_{j}=-\frac{3}{2 j} d_{j-1} .
$$


On the other hand, from the equation $(2 k)$ of $\left(L_{m}\right)$ with $k$ replaced by $j$ we see that

$$
(2 j+3) d_{j}=3 \widetilde{c}_{j} .
$$

Thus we have

$$
d_{j}=\frac{3}{2 j+3} \widetilde{c}_{j} .
$$

It follows from (4.6) and (4.7) that

$$
d_{j}=-\frac{3}{2 j+3} \frac{3}{2 j} d_{j-1}=-\frac{3^{2}}{2 j(2 j+3)} d_{j-1}
$$

for $j=2, \cdots, m$, which implies that

$$
d_{j}=(-1)^{j} \frac{3^{2 j}}{5 \cdot 7 \cdot 9 \cdots(2 j+3) 2^{j}(j !)},
$$

where we should note that $d_{1}=(-9) /(5 \cdot 2)$. We thus obtain (4.3) by setting $j=m$ in (4.8).

Theorem 4.2. For each $m \in \mathbb{N}$, we have

$$
\mathcal{R}_{m}=\left\{1,\left(\frac{5}{3}\right)^{2},\left(\frac{7}{3}\right)^{2}, \cdots,\left(\frac{2 m+3}{3}\right)^{2}\right\} .
$$

Proof. We prove the theorem by induction on $m$. As was pointed out in Remark 3.3, we only have to show that

$$
\left(\frac{2 m+3}{3}\right)^{2} \in \mathcal{R}_{m}
$$

for every $m \geq 2$, assuming that (4.9) with $m$ replaced by $m-1$ is true.

Let us recall that

$$
b_{0} b_{m}-a_{m}=b_{0}^{2} q_{m}\left(b_{0}^{2}\right)-p_{m}\left(b_{0}^{2}\right)=P_{m}\left(b_{0}^{2}\right),
$$

where $p_{m}(t)$ and $q_{m}(t)$ are polynomials of degree $m$. It follows from (4.11) that $d_{m}$ is equal to the coefficient of $t^{m+1}$ of the polynomial $P_{m}(t)$, of which degree is $m+1$. Also it follows from (4.11) that the constant coefficient of $P_{m}(t)$ is given by $-c_{m}$.

By hypothesis of the induction, we have

$$
\mathcal{R}_{m-1}=\left\{1,\left(\frac{5}{3}\right)^{2},\left(\frac{7}{3}\right)^{2}, \cdots,\left(\frac{2 m+1}{3}\right)^{2}\right\} .
$$

Since $\mathcal{R}_{m-1} \subset \mathcal{R}_{m}$ by Proposition 3.2 , we see that

$$
1,\left(\frac{5}{3}\right)^{2},\left(\frac{7}{3}\right)^{2}, \cdots,\left(\frac{2 m+1}{3}\right)^{2}
$$

are the roots of $P_{m}(t)$. For simplicity, we put

$$
\lambda_{j}=\left(\frac{2 j+1}{3}\right)^{2} \quad(j=1,2, \cdots, m) .
$$

Since there exists one more root $\lambda \in \mathcal{R}_{m}$ of $P_{m}(t)$, we find that

$$
P_{m}(t)=d_{m}\left(t-\lambda_{1}\right)\left(t-\lambda_{2}\right) \cdots\left(t-\lambda_{m}\right)(t-\lambda) .
$$


Noting that $P_{m}(0)=-c_{m}$, we get

$$
d_{m}(-1)^{m+1} \lambda_{1} \lambda_{2} \cdots \lambda_{m} \lambda=-c_{m} .
$$

Hence, by using (4.2), (4.3) and (4.13), we obtain

$$
(-1)^{m+1}\left[\prod_{j=1}^{m}\left(\frac{2 j+1}{3}\right)^{2}\right] \lambda=-\frac{c_{m}}{d_{m}}=(-1)^{m+1} \prod_{j=1}^{m+1}\left(\frac{2 j+1}{3}\right)^{2} .
$$

Therefore we can conclude that

$$
\lambda=\left(\frac{2 m+3}{3}\right)^{2}
$$

which implies (4.10).

For each $m \in \mathbb{N}$, the polynomial (4.11) has $2 m+2$ roots:

$$
b_{0}= \pm 1, \pm \frac{5}{3}, \cdots, \pm \frac{2 m+3}{3} .
$$

If we choose the root $b_{0}=+(2 j+1) / 3$ for a fixed $j$ with $1 \leq j \leq m+1$, then we can define $a_{1}, \cdots, a_{m}, b_{1}, \cdots, b_{m}$ by Proposition 2.4. With these $a_{1}, \cdots, a_{m}, b_{1}, \cdots, b_{m}$ obtained, we construct $\psi_{j,+}^{(m)}(x)$ by (2.2). It follows from Propositions 2.1 and 1.2 that $\psi_{j,+}^{(m)}$ is a zero mode of the magnetic Dirac operator $H_{A_{j,+}^{(m)}}:=\sigma \cdot\left(D-A_{j,+}^{(m)}\right)$, where $A_{j,+}^{(m)}$ is defined by (1.3) with

$$
h(x)=\frac{2 j+1}{\langle x\rangle^{2}}, \quad \psi(x)=\psi_{j,+}^{(m)}(x) .
$$

The sequence $\left\{\psi^{(m)}\right\}_{m=1}^{\infty}$ constructed in Adam, Muratori and Nash [1] is now obtained by putting

$$
\psi^{(m)}(x):=\psi_{m+1,+}^{(m)}(x) .
$$

We make a few of concluding remarks.

(i) For each $m \in \mathbb{N}$, set

$$
\Psi_{m}=\left\{\psi_{j,+}^{(m)}(x) \mid j=1,2, \cdots, m+1\right\} .
$$

Adam, Muratori and Nash [1] pointed out that

$$
\Psi_{1} \varsubsetneqq \Psi_{2} \varsubsetneqq \cdots \varsubsetneqq \Psi_{m} \varsubsetneqq \cdots \text {. }
$$

(ii) In a similar manner, choosing the root $b_{0}=-(2 j+1) / 3$, we can construct a different sequence of zero modes from $\left\{\psi^{(m)}\right\}_{m=1}^{\infty}$ defined above.

Acknowledgments. T.U. would like to thank Ryuichi Ashino for his help with our Maple program, and thank Takeshi Usa for his valuable comment on the solvability of the Adam-Muratori-Nash polynomials that Galois theory seems to play an important role behind the scene. 


\section{REFERENCES}

[1] C. Adam, B. Muratori and C. Nash, Zero modes of the Dirac operator in three dimensions, Phys. Rev. D 60 (1999), 125001-1 - 125001-8.

[2] C. Adam, B. Muratori and C. Nash, Degeneracy of zero modes of the Dirac operator in three dimensions, Phys. Lett. B 485 (2000), 314-318

[3] C. Adam, B. Muratori and C. Nash, Multiple zero modes of the Dirac operator in three dimensions, Phys. Rev. D 62 (2000), 085026-1 - 085026-9.

[4] A.A. Balinsky and W.D. Evans, On the zero modes of Pauli operators, J. Funct. Analysis, 179 (2001), 120-135.

[5] A.A. Balinsky and W.D. Evans, On the zero modes of Weyl-Dirac operators and their multiplicity, Bull. London Math. Soc., 34 (2002), 236-242.

[6] A.A. Balinsky and W.D. Evans, Zero modes of Pauli and Weyl-Dirac operators, Advances in differential equations and mathematical physics (Birmingham, AL, 2002), 1-9, Contemp. Math., 327, Amer. Math. Soc., Providence, Rhode Island, 2003.

[7] A.A. Balinsky, W.D. Evans and T. Umeda, The Dirac-Hardy and Dirac-Sobolev inequalities in $L^{1}$, to appear in Publ. Res. Inst. Math. Sci. Kyoto Univ.

[8] H.M. Edwards, The construction of solvable polynomials, Bull. Amer. Math. Soc. 46 (2009), $397-412$.

[9] D.M. Elton, New examples of zero modes, J. Phys. A: Math. Gen. 33 (2000), 7297-7303.

[10] D.M. Elton, The local structure of zero mode producing magnetic potentials, Commun. Math. Phys. 229 (2002), 121-139.

[11] L. Erdös and J.P. Solovej, The kernel of Dirac operators on $\mathbb{S}^{3}$ and $\mathbb{R}^{3}$, Rev. Math. Phys. 13 (2001), 1247-1280.

[12] J. Fröhlich, E.H. Lieb and M. Loss, Stability of Coulomb systems with magnetic fields. I. The one-electron Atom, Commun. Math. Phys. 104 (1986),251-270.

[13] M. Loss and H.T. Yau, Stability of Coulomb systems with magnetic fields. III. Zero energy bound states of the Pauli operators, Commun. Math. Phys. 104 (1986), 283-290.

[14] P. Pickl, Generalized eigenfunctions for critical potentials with small perturbations, J. Math. Phys. 48 (2007), 123505-1 - 123505-31.

[15] P. Pickl and D. Dürr, On adiabatic pair creation, Commun. Math. Phys. 282 (2008), 161-198.

[16] P. Pickl and D. Dürr, Adiabatic pair creation in heavy ion and laser fields, Europhys. Lett. 81 (2008), 40001-40007.

[17] Y. Saitō and T. Umeda, Eigenfunctions at the threshold energies of magnetic Dirac operators, to appear in Rev. Math. Phys.

[18] Wikipedia, Galois Theory, http://en.wikipedia.org/wiki/Galois_theory. 